\title{
Optical Fiber Imaging Based Tomographic Reconstruction of Burner Flames
}

\author{
Md. Moinul Hossain, Member, IEEE, Gang Lu, Senior Member, IEEE, and Yong Yan, Fellow, IEEE
}

\begin{abstract}
This paper presents the design, implementation, and evaluation of an optical fiber imaging based tomographic system for the 3-D visualization and characterization of a burner flame. Eight imaging fiber bundles coupled with two RGB chargecoupled device cameras are used to acquire flame images simultaneously from eight different directions around the burner. The fiber bundle has 30k picture elements and an objective lens with a $92^{\circ}$ angle of view. The characteristic evaluation of the imaging fiber bundles and the calibration of the system were conducted to ensure the accuracy of the system. A new tomographic algorithm that combines the logical filtered back-projection and the simultaneous algebraic reconstruction technique is proposed to reconstruct the flame sections from the images. A direct comparison between the proposed algorithm and other tomographic approaches is conducted through computer simulation for different test templates and numbers of projections. The 3-D reconstruction of the cross- and longitudinal-sections of a burner flame from image projections obtained from the imaging system was also performed. The effectiveness of the imaging system and computer algorithm is assessed through experimental tests.
\end{abstract}

Index Terms-Charge-coupled device (CCD) camera, flame, imaging fiber, luminosity distribution, three-dimensional reconstruction, tomography.

\section{INTRODUCTION}

$\mathbf{T}$ HREE-DIMENSIONAL (3-D) monitoring and characterization of burner flames plays an important part in the in-depth understanding and subsequent optimization of fossilfuel-fired combustion processes [1], [2]. Among the techniques developed for the 3-D flame visualization, passive optical tomography has shown clear advantages over other techniques [3], [4], including the simple system setup, high spatial resolution, fast system response, and relatively low cost [1], making it most suitable for the 3-D reconstruction and characterization

Manuscript received June 20, 2011; revised October 4, 2011; accepted January 4, 2012. Date of publication March 5, 2012; date of current version April 6, 2012. This work was supported by the Research Councils U.K. (RCUK)'s Energy Program under Grant EP/G062153/1, but the views expressed are those of the authors and not necessarily those of the RCUK. The Energy Program is an RCUK cross-council initiative led by the Engineering and Physical Sciences Research Council and contributed to by the Economic and Social Research Council, Natural Environment Research Council, Biotechnology and Biological Sciences Research Council, and Science and Technology Facilities Council. The Associate Editor coordinating the review process for this paper was Dr. Jiong Tang.

The authors are with the Instrumentation, Control and Embedded Systems Research Group, School of Engineering and Digital Arts, University of Kent, CT2 7NZ Canterbury, U.K. (e-mail: mmh22@kent.ac.uk; g.lu@kent.ac.uk; y.yan@kent.ac.uk).

Color versions of one or more of the figures in this paper are available online at http://ieeexplore.ieee.org.

Digital Object Identifier 10.1109/TIM.2012.2186477 of flames in practical furnaces. Both single camera and multiple cameras based tomographic systems have been reported [1], [2]. Torniainen et al. [5] used a tomographic inversion method to study unsteady reacting flows, where numerical simulations were performed to evaluate the proposed method for reconstructing the 2-D fuel concentration distributions from a numerical simulation of a turbulent reacting square jet. Fisher and Burkhardt [6] first proposed a multispectral tomographic imaging system for the 3-D temperature measurement of a candle flame. Brisley et al. [7] demonstrated a single-camera system for the 3-D temperature measurement of a gaseous flame. Huang et al. [8] also proposed a stereoscopic imaging based single camera system for the reconstruction of soot temperature and volume fraction profiles of a flame. Zhou et al. [9] developed a single charge-coupled device (CCD) camera system based on optical sectioning tomography to reconstruct the 3-D distribution of candle flame temperature. Although the single camera approach is simple in structure and low in cost and can be installed on a practical furnace, it can only be applied under strict conditions where the flame has a high level of rotational symmetry. It is therefore unsuitable for monitoring of unsteady turbulent flames.

A number of multi-camera systems have been reported for 3-D flame reconstructions. Bheemul et al. [10] developed a three-camera system for 3-D visualization and characterization of gas-fired flames, where three 2-D images captured around the flame were used to build the flame geometrical model from which a set of characteristic parameters is also determined. Ishino et al. [11], [12] developed a multi-lens camera system to obtain instantaneous 3-D gray-scale distributions of a propane turbulent flame [11] and also accomplished the computerized tomography reconstruction technique for measuring an instantaneous 3-D distribution of chemiluminescence of a turbulent premixed flame [12]. The system has a total of 40 special camera lenses arranged on a semi-circle on one side of the burner. However, the system proposed is single shot and film based, and thus, it is unsuitable for taking multiple shots of a turbulent flame. Zhou et al. [13] performed numerical investigations into the 3-D visualization and temperature distribution in large-scale boiler furnaces, where eight CCD cameras were assumed to be mounted around the furnace to capture radiative energy images inside the furnace and to simulate the 3-D temperature distribution. However, a number of difficulties were encountered, such as synchronization of all of the eight cameras. Floyd et al. proposed tomographic techniques for the high-resolution and instantaneous 3-D reconstruction of the chemiluminescence intensities from $\mathrm{CH}^{*}$ in the reaction zone of matrix burner flames [14] and a premixed turbulent opposed jet flame [15]. The 
high-resolution reconstruction was achieved using 48 views of a rotating CCD camera, while the instantaneous reconstruction was performed using ten instantaneous images from five cameras with a set of mirrors. The perspective distortion due to real lens effects (i.e., focus, beam divergence, and out-ofplane effects) was corrected to achieve full 3-D reconstructions [14]. Anikin et al. [16] described a tomographic system to detect the 2-D distribution of the $\mathrm{OH}^{*}$ chemiluminescence in the reaction zone of turbulent flames. The system employed ten Kepler telescopes, each conjoined with an optical fiber bundle of 90 individual fibers arranged in a single row, to capture the light of flame from different angles and to transmit onto a single CCD camera. The telescope has an iris aperture positioned in the focal plane of the objective lens which guarantees that only parallel rays can pass the system. Schwarz [17] proposed a Schlieren imaging based multi-tomographic technique for the 3-D visualization of a gaseous flame. Wan et al. [18] introduced an online emission spectral tomography system with a four channel video signal processor to test the 3-D distribution parameters of fluid fields. Ihrke et al. [19] reported image-based tomography method for reconstructing a volumetric model from multiple images of fire, and an eightcamera setup was used to acquire the images. Moratti et al. [20] described a digital imaging system for the 3-D reconstruction of an industrial flame, where the flame volume and surface area were determined. Gilabert et al. [21] developed a threecamera tomographic system where three red, green, and blue (RGB) CCD cameras, together with dedicated optical transmission units, were used to capture concurrently six equiangular 2-D images of a flame. A tomographic algorithm which combined the logical filtered back-projection (LFBP) and algebraic reconstruction technique (ART) was developed for the 3-D reconstruction of the luminosity distribution of flames from the six image projections. Despite the obvious improvement in the reconstruction of flames from multiple projections, the number of projections available (only six) is still too few for a higher spatial resolution. In addition, the complexity of the system makes it unsuitable for installation on a large-scale furnace.

Despite various advances in 3-D flame tomographic systems, a range of technical challenges remain to be resolved. In particular, a practical hardware platform for the generation of a sufficient number of image projections to achieve improved spatial resolution and reliability of the system is desirable. The general descriptions of the 3-D imaging system and the methodology, together with the preliminary results, were reported at the 2011 IEEE International Instrumentation and Measurement Technology Conference [22]. This paper presents a detailed description of the system design, implementation, and evaluation of an imaging fiber based tomographic system for the $3-D$ visualization and characterization of burner flames in a practical furnace. The system uses eight high-specification imaging fiber bundles coupled with two CCD cameras to produce simultaneously eight image projections around a flame. Characteristic evaluations of the fiber bundles and the system calibration have been conducted to ensure the system accuracy and reliability. A new tomographic approach that combines the LFBP and the simultaneous ART (LFBP-SART) is proposed for the reconstruction of flame sections. A direct comparison

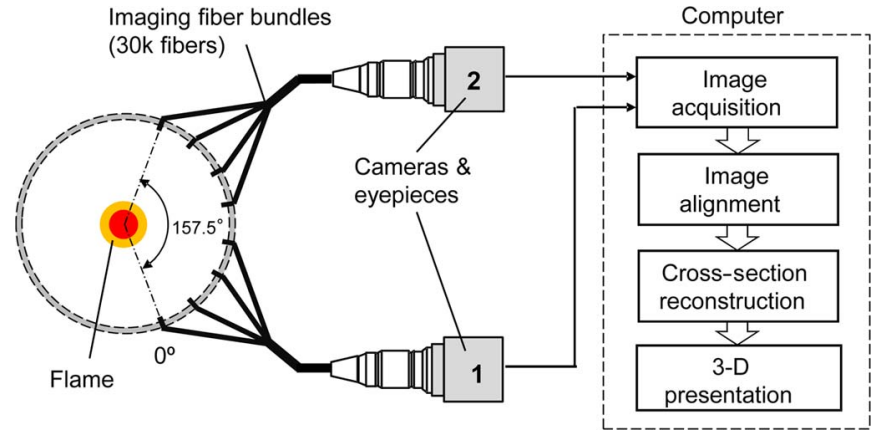

Fig. 1. Schematic of the 3-D flame imaging system.

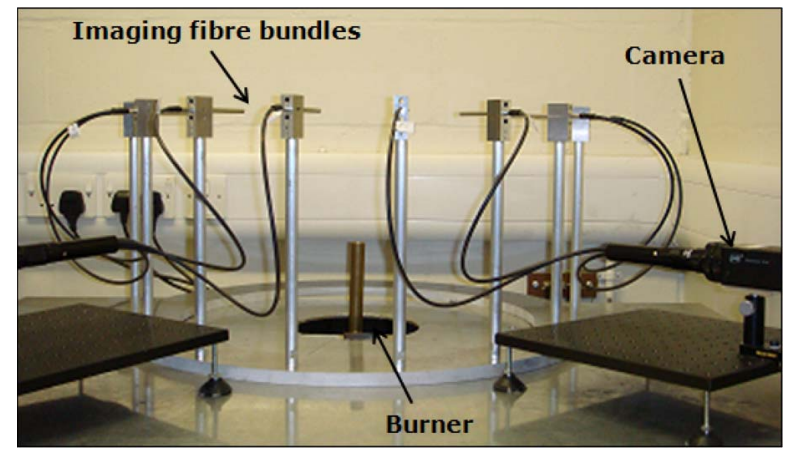

Fig. 2. Physical implementation of the 3-D flame imaging system.

among the different algorithms has been carried out in terms of resolution and accuracy for different numbers of projections. Simulation results are presented for the 3-D reconstruction of the luminosity distribution of flame cross-sections and longitudinal-sections of flame.

\section{SySTEM DESCRIPTION}

\section{A. System Setup}

Fig. 1 shows a schematic diagram of the 3-D tomographic flame imaging system. The system consists of eight imaging optical fiber bundles with associated coupling lenses, two RGB CCD cameras, two frame grabbers, and a personal computer with application software. The flexible imaging fiber bundles, each having a total of 30000 individual optical fibers, are $650 \mathrm{~mm}-700 \mathrm{~mm}$ long, with an outer diameter of $4 \mathrm{~mm}$ and a $92^{\circ}$ objective lens. Four of the eight fiber bundles are joined onto a single eyepiece/coupling lens, forming four flame images into the same CCD camera. The eight imaging fiber bundles are arranged on one side of the burner as a semi-circle and are fixed in positions on eight aluminum stands with a separation angle of $22.5^{\circ}$ between two adjacent fiber bundles. The radial distance from the objective lens of the fiber (to the burner axis, i.e., objective distance) is $250 \mathrm{~mm}$. The camera has an RGB color progressive 1/3 inch CCD sensor with square pixels and a primary mosaic filter, and a frame rate of 25 frames/s. Each camera has a resolution of $1024 \times 768$ pixels. The two frame grabbers are synchronized by the system software to ensure the acquisition of the image signals simultaneously. With such an arrangement, the imaging system is capable of producing a total of eight flame images concurrently from eight different directions around the flame. Fig. 2 shows the physical implementation of the 3-D flame imaging system. 
TABLE I

Averaged Gray Intensities of the LED IMAges TAKEn Using EIGHT Fiber BundLES For Different Voltages

\begin{tabular}{|c|c|c|c|c|c|c|c|c|}
\hline \multirow{2}{*}{$\begin{array}{c}\text { LED } \\
\text { input (V) }\end{array}$} & \multicolumn{7}{|c|}{ Averaged gray intensities of the LED images } \\
\cline { 2 - 9 } & Fiber 1 & Fiber 2 & Fiber 3 & Fiber 4 & Fiber 5 & Fiber 6 & Fiber 7 & Fiber 8 \\
\hline 2.80 & 13 & 13 & 14 & 12 & 12 & 12 & 13 & 12 \\
\hline 3.08 & 82 & 80 & 80 & 82 & 81 & 81 & 81 & 81 \\
\hline 3.45 & 199 & 198 & 198 & 199 & 200 & 199 & 199 & 200 \\
\hline 3.75 & 211 & 210 & 210 & 211 & 212 & 211 & 211 & 212 \\
\hline 4.00 & 218 & 218 & 218 & 220 & 221 & 220 & 220 & 221 \\
\hline 4.30 & 224 & 222 & 223 & 223 & 224 & 224 & 224 & 224 \\
\hline
\end{tabular}

\section{B. Characteristic Evaluation of the Imaging Fiber Bundles}

To achieve reliable and accurate image acquisition and subsequent 3-D reconstruction of the flame, it must be ensured that the images transmitted through the eight imaging fibers have identical geometric and luminous characteristics for a given reference. Differences between the images result from a number of factors, including the dissimilarity between the physical properties of the eight optical fiber bundles and imaging sensors, the dissimilar settings of the two cameras, and the misaligned locations of the fiber bundles. The characteristics of the imaging fibers and the cameras have therefore to be evaluated. Luminosity evaluations were performed using a lightemitting diode (LED) white backlight panel as a reference light source. The different light intensities of the LED were achieved by varying the voltages supplied. For each lighting condition, a total of ten images were captured using the eight fiber bundles, respectively, the averaged gray intensities were calculated for each fiber bundle, and the differences of the gray intensities were analyzed. Table I illustrates the averaged gray intensities of the images taken through the eight fiber bundles for different LED light settings. It can be seen that the images of the eight fiber bundles have very similar intensities with a maximum discrepancy no greater than 2 gray-levels in the range from 13 to 224 .

The image brightness attenuation due to the vignetting effect of the optical components was evaluated using the LED backlight panel. The brightness attenuation was checked over the effective image area of $128 \times 256$ pixels with reference to the gray-level of the center point of the image. It was found that the normalized standard deviation of the gray-levels over the effective image area is less than $4 \%$ at an objective distance of $250 \mathrm{~mm}$.

The radial distortion of the images due to the imaging components has also been investigated. This is particularly important for image acquisitions where a wide-angle lens is used with each fiber bundle. The evaluation was conducted using a square grid template placed at the center of the burner. Images of the template were captured by the imaging fiber bundles from different objective distances. The radiuses of the captured images were computed from the center of the template image, and the normalized radial errors were computed, as shown in Fig. 3. It can be seen that the normalized radial error

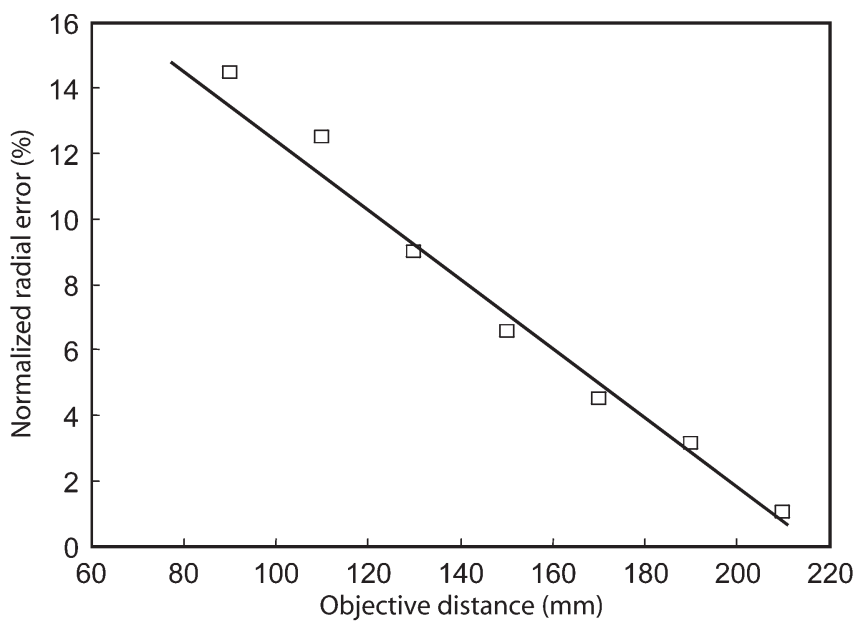

Fig. 3. Normalized radial error for different objective distances.

decreases with the objective distance and is less than $2 \%$ when the objective distance is $210 \mathrm{~mm}$ or greater.

\section{System Calibration}

The calibration of the system was conducted by reproducing the geometrical relationship between the eight fiber bundles and the flame. A geometric alignment was undertaken to ensure that the optical paths of the eight fiber bundles pass through the same reference line, i.e., burner central line. This was achieved by using a template which is a circular rod with several adjacent reference marks. The template was placed where the burner was located. A computer program was designed to create an image window with a number of reference lines. The positions of the fiber bundles were then adjusted so that the template images from the eight fiber bundles matched the same reference lines.

The accuracy of the dimensional measurement of the system was evaluated using a similar template. The results obtained are shown in Fig. 4, where each of the data point is an average of ten readings. A diagonal straight line is also plotted to give a direct comparison between the measured results and the reference values. The maximum deviation of the measured length from the reference length is less than $2 \%$. The repeatability of the system was also evaluated by recording consecutive readings for a given reference length. For instance, the normalized standard deviation of the ten readings for a reference length of 


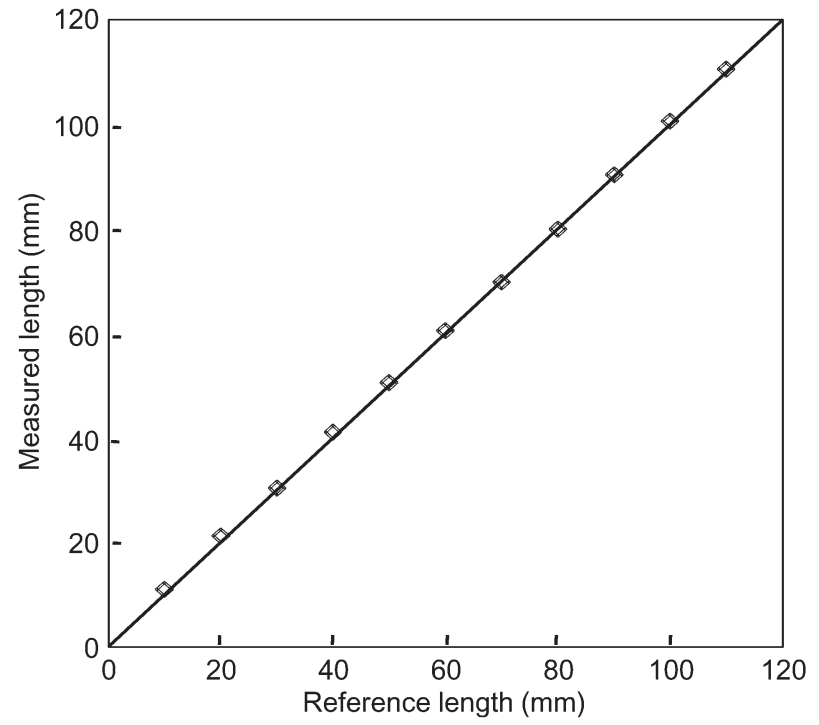

Fig. 4. Comparison between the measured length and the reference length.

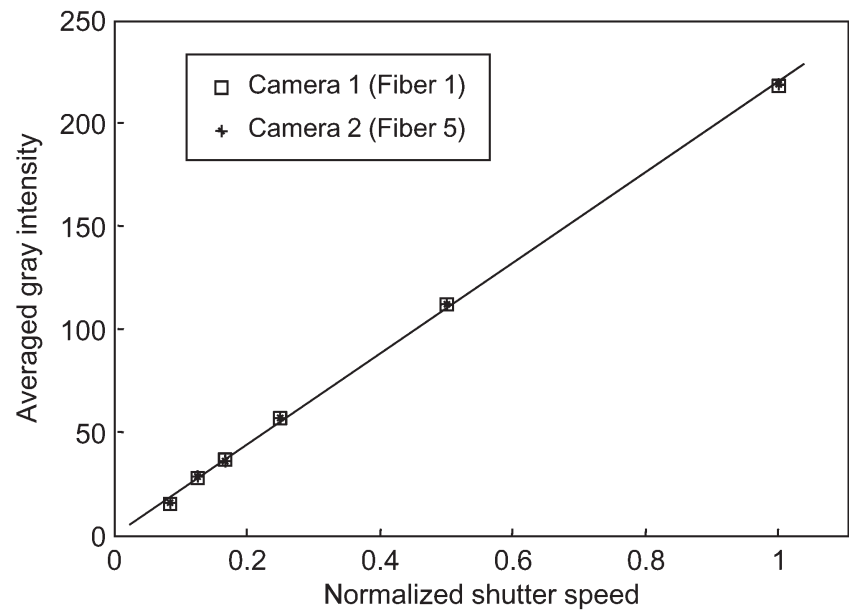

Fig. 5. Averaged gray intensities of the LED images for different shutter speeds of the cameras.

$110 \mathrm{~mm}$ was $0.54 \%$, equivalent to $0.60 \mathrm{~mm}$ along the longitudinal direction.

The brightness variation of the captured images with the camera shutter speed was also evaluated using the LED backlight panel. Fig. 5 shows the averaged gray intensities of the LED images taken by two fiber bundles of the two cameras for different camera shutter speeds. The shutter speed is normalized to its maximum value at which the image is approaching saturation. It has been found that the imaging fiber bundles have very similar sensitivities for the same shutter speed with the maximum normalized standard deviation of $2.5 \%$. In addition, the brightness variation of the captured images with the objective distance was also evaluated using the same light source, and it was found that the fiber bundles have consistent sensitivities, with the maximum normalized standard deviation of $1 \%$ for the tested objective distance between 130 and $280 \mathrm{~mm}$.

\section{TOMOgRAPhic Reconstruction Algorithms}

Various computed tomography algorithms have been developed for the 3-D reconstructions for different applications.

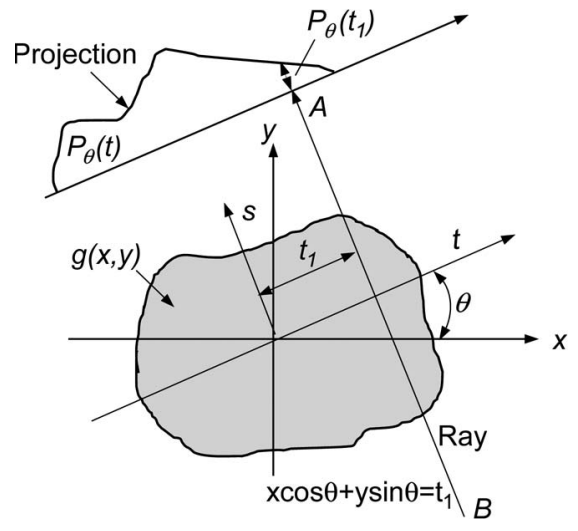

Fig. 6. Schematic of the 2-D Radon transform.

Among those, filtered back-projection (FBP), LFBP, and ART are mostly used for the reconstructions of fluid and thermal dynamic media such as multi-phase flows and combustion flames [23], [24]. Theoretically, tomographic systems working on those algorithms require a sufficient number of projections to achieve a high spatial resolution of reconstructions. This often results in great system complexity, long response time, and high capital cost, particularly for passive optical tomographic systems. Gilabert et al. [21] used only six available image projections for the reconstruction of flame sections through the combined LFBP and ART algorithm (LFBP-ART) which can effectively eliminate noises caused by a limited number of image projects. The LFBP-ART has been further improved by introducing two more image projections into the reconstruction [22]. In the present work, a new tomographic algorithm which relies on the combination of the LFBP and the simultaneous ART, i.e., LFBP-SART, is proposed. The LFBP-SART has shown a possibility of using a few image projections for improved 3-D reconstruction of flames for an acceptable resolution. To examine the impact of the number of projections on the quality of the reconstruction so as to verify the suitable number of imaging sensors which the system requires, both theoretical study and computer simulation of the tomographic reconstructions have been conducted.

\section{A. LFBP Algorithms}

The LFBP is a modified algorithm of the FBP [25], which is based on the projection slice theorem and is used to implement the inverse Radon transform [23]. As shown in Fig. 6, assuming that $g(x, y)$ is a 2-D section function of an object, $P_{\theta}\left(t_{1}\right)$ is the integral function of $g(x, y)$ along straight line $x \cos \theta+$ $y \sin \theta=t_{1}$, which is also known as the Radon transform of $g(x, y)$ [23]; the FBP reconstruction function of $g(x, y)$ can be approximated to [21], i.e.,

$$
\begin{aligned}
& g(\bar{x}) \approx \frac{\pi}{M_{\text {proj }}} \sum_{i=1}^{M_{\text {proj }}} Q_{\theta i}(\bar{x} \cdot \bar{n}), \\
& Q_{\theta_{i}}(\bar{x} \cdot \bar{n})=\left(h * P_{\theta}\right)\left(x^{\prime}\right),
\end{aligned}
$$

where $Q_{\theta i}$ is the filtered projection, $P_{\theta}$ is the projection of $g(\bar{x})$ along angle $\theta$ and through the rectilinear path determined by the Dirac delta function $\delta\left(\overline{\mathrm{x}} \cdot \overline{\mathrm{n}}-\mathrm{x}^{\prime}\right), h$ is the filter impulse 
response, $*$ denotes convolution, $M_{\text {proj }}$ is the total number of projections, $\bar{n}=(\cos \theta, \sin \theta)$ is a unit vector normal to the projection beam, and $\bar{x}$ is a vector on the projection beam. The difference between the FBP and the LFBP lies in the transformation of (1), where the additive operation $\Sigma$ is substituted by the logical operator $\Theta$ in such a way that if $A, B$, and $C$ are square matrices [22], i.e.,

$$
C=A \Theta B \quad c_{i j}= \begin{cases}0, & \text { if } a_{i j}=0 \cup b_{i j}=0 \\ 1, & \text { if } a_{i j}=b_{i j} .\end{cases}
$$

Rewriting (1) to the LFBP form,

$$
g(\bar{x}) \approx \frac{\pi}{M_{\text {proj }}} \stackrel{M_{\text {proj }}}{\Theta} Q_{\theta i}(\bar{x} \cdot \bar{n}) .
$$

\section{B. SART Algorithms}

The SART approach is a combination of ART and simultaneous iterative reconstruction technique (SIRT) algorithms that can give reconstruction of good quality and numerical accuracy with only one iteration [23]. In the ART, the image cross-sections are considered to be composed of an array of unknowns. The concept of "ray" is introduced in the ART, where a ray is considered to be a "thick" line running across the cross-sections. The problem can then be reduced to solve a set of simultaneous equations [21], i.e.,

$$
\begin{gathered}
\omega_{11} g_{1}+\omega_{12} g_{2}+\cdots+\omega_{1 N} g_{N}=p_{1}, \\
\vdots \\
\omega_{M 1} g_{1}+\omega_{M 2} g_{2}+\cdots+\omega_{M N} g_{N}=p_{M},
\end{gathered}
$$

where $\omega_{N M}$ is a weighting factor, $g_{j}$ denotes the constant value of $g(x, y), p_{i}$ is the portion of the projection, $M$ is the total number of rays, and $N$ is the total number of cells. Initial vector solution $\bar{g}^{(0)}=\left(g_{1}^{(0)}, g_{2}^{(0)}, \ldots, g_{N}^{(0)}\right.$ is guessed by assigning a value of zeros, and successive values of vector solution are solved by,

$$
\bar{g}^{(i)}=\bar{g}^{(i-1)}-\frac{\left(\bar{g}^{(i-1)} \cdot \bar{\omega}_{i}-p_{i}\right)}{\overline{\omega_{i}} \cdot \bar{\omega}_{i}} \bar{\omega}_{\mathrm{i}}
$$

where $\bar{\omega}_{\mathrm{i}}=\left(\omega_{\mathrm{i} 1}, \omega_{\mathrm{i} 2}, \ldots, \omega_{\mathrm{iN}}\right)$ and $\bar{\omega}_{\mathrm{i}} \cdot \bar{\omega}_{\mathrm{i}}$ is the dot product of $\bar{\omega}_{\mathrm{i}}$ with itself [21]. More details about the ART algorithms are available in [23], [26], and [27].

SIRT [23] is an effective method to solve inverse problems in an iterative way, and at the expense of slower convergence, SIRT usually leads to better reconstruction results than ART. The convergence of SIRT has been established [28]. The main features of SART also include reduced errors in the approximation of ray integrals of a smooth image by finite sums, simultaneously updating weights, and heuristic procedure for the improved quality of reconstructions. A series of steps has been taken for SART implementation. First, a sequential implementation can be carried out by using the update equation of (6) and expressed in terms of SART symbols,

$$
\overline{\mathrm{g}}^{(k+1)}=\bar{g}^{(k)}+\bar{\omega}_{i} \frac{p_{i}-\bar{\omega}_{i}^{T} \bar{g}^{(k)}}{\bar{\omega}_{i}^{T} \bar{\omega}_{i}},
$$

where $\overline{\omega_{i}}$ denotes the $i$ th row vector of the array $\omega_{\mathrm{ij}}, \bar{g}^{(k)}$ is the estimate image vector, and $k$ is the number of iterations.
Then, simultaneously applying to a pixel on bilinear elements and the average of the corrections generated by all the rays in a projection, instead of sequential updating pixels on a ray-by ray basis for superior results, it can be stated as follows [23],

$$
g_{j}^{(k+1)}=g_{j}^{(k)}+\frac{\sum_{i}\left[\omega_{i j} \frac{p_{i}-\bar{\omega}_{i}^{T} \bar{g}^{(k)}}{\sum_{j=1}^{N} \omega_{i j}}\right]}{\sum_{i} \omega_{i j}}, \quad(i=1,2, \ldots, M)
$$

where the summation with respect to $i$ is over the rays intersecting the $j$ th image elements for a given direction and $M$ is the total number of rays.

\section{LFBP-SART Algorithms}

The task of performing the tomographic reconstruction of the luminosity distribution of the flame presents a major challenge in that the number of independent projections is limited, leading to an underdetermined problem [21]. However, this can be overcome using the LFBP-SART technique. The advantages of the LFBP-SART over the LFBP-ART are reduced errors and faster convergence. It was proven that the LFBP can effectively restrain the blurring in the reconstruction, which is normally encountered in the FBP [25]. Another advantage of the LFBP over the FBP is that it provides the information of outer contours of the object being reconstructed, and this information can be utilized to increase the number of equations used in the SART, i.e.,

$$
\omega_{i j} g_{j}=0, \quad \text { if } \quad c_{i j}=0, \quad\left\{c_{i j} \in C\right\}
$$

where $w_{i j}$ and $g_{j}$ are the elements in (5) and $C$ is the matrix in (4). This additional set of equations corrects the hyperplanes represented in (5), resulting in a more accurate solution in performing the simultaneous update operation described in (8). The external contour obtained using the LFBP is utilized to determine the points that have to be calculated using the SART. This approach significantly reduces the number of required calculations and is also applicable where the projection angle is large. The stepwise implementation procedure of the LFBPSART algorithms can be briefly described as follows:

1) Get the 2-D images matrices, generation, and centralization of projections.

2) Carry out the filtering and back-projection by (1) and (2).

3) Apply (3) and (4) to compute the LFBP algorithm.

4) Determine the external contour.

5) Identify the positions that have to be calculated by the SART using (9).

6) Apply the SART (8) algorithm for integration.

7) Generate the flame sections, reallocation of sections, and data presentation.

It has been realized that the tomographic reconstruction algorithms as discussed previously are based on an orthographic imaging model, i.e., all pixel's rays are assumed to be parallel to the optical axis of a particular view. This is not the case in the present optical setup, particularly considering the wide-angle 


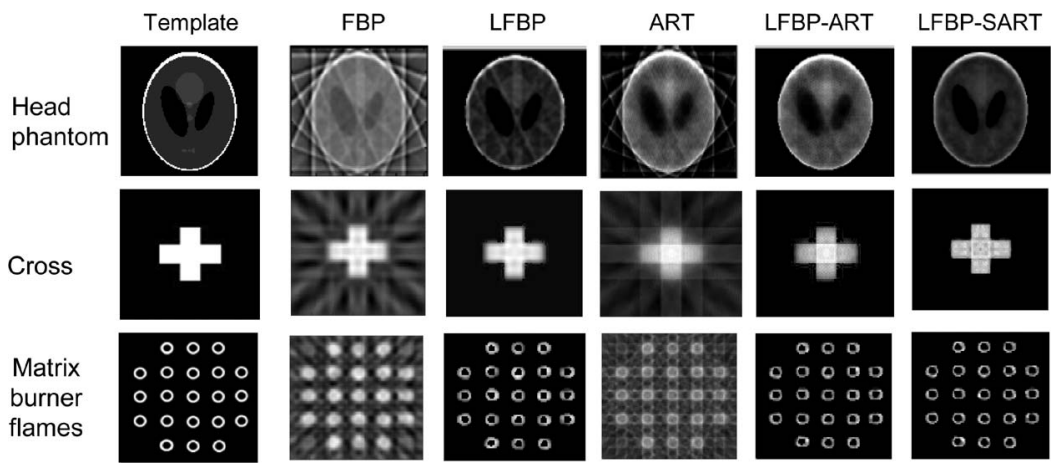

Fig. 7. Comparison of the five tomographic algorithms for eight projections.

$\left(92^{\circ}\right)$ objective lenses are used. The inconsistency between the imaging model and the optical setup would introduce errors in the reconstruction of the flame. The problem can be more serious in the regions which are away from the optical axis since the impact of the rays passing at an angle through those parts of the flame cannot be neglected. This is a drawback of the photography based tomographic reconstruction through the conventional back-projection [12]. In practice, the problem can be minimized by using a relatively small flame in comparison to the objective distance. In addition, the optical axes of the fiber bundles should be aligned to the region of most interest, i.e., the root part of the flame (the tip part of the flame is less important from a combustion point of view). To fully solve the problem, however, full-fledged 3-D reconstruction algorithms should be implemented [14].

\section{RESUlts AND Discussions}

\section{A. Computer Simulation}

To examine the effectiveness of the LFBP-SART as compared to other tomographic algorithms for different numbers of projections, computer simulation was undertaken for the reconstruction of 8-bit gray-level template images with a resolution of $256 \times 256$. Five algorithms were implemented for different numbers of projections. Fig. 7 shows the simulation results using the FBP, ART, LFBP, LFBP-ART, and LFBP-SART for different test templates (i.e., a head phantom and a cross [21]) and a matrix of burner flames [14]. For each test, eight different 1-D projections that were taken on an equiangular basis and the angle between the first and the last projections have to be $180^{\circ}$ at the maximum; the case of using eight projections gives the equiangular angle of $22.5^{\circ}$ between two consecutive projections. It can be seen that, with only eight projections, the LFBP-SART gives a better reconstruction result over all other algorithms, with a significant reduction of noise within the reconstructed images.

The accuracy of the reconstruction results was further assessed by using two evaluation criteria, i.e., correlation coefficient (CC) and peak signal-to-noise ratio (PSNR), which were computed between the original images of the templates and the reconstructed results. Figs. 8 and 9 show the $\mathrm{CC}$ and the PSNR against the number of projections of the head phantom, respectively. It is clear that the LFBP-SART algorithm provides a better CC and the highest PSNR. It can be observed from

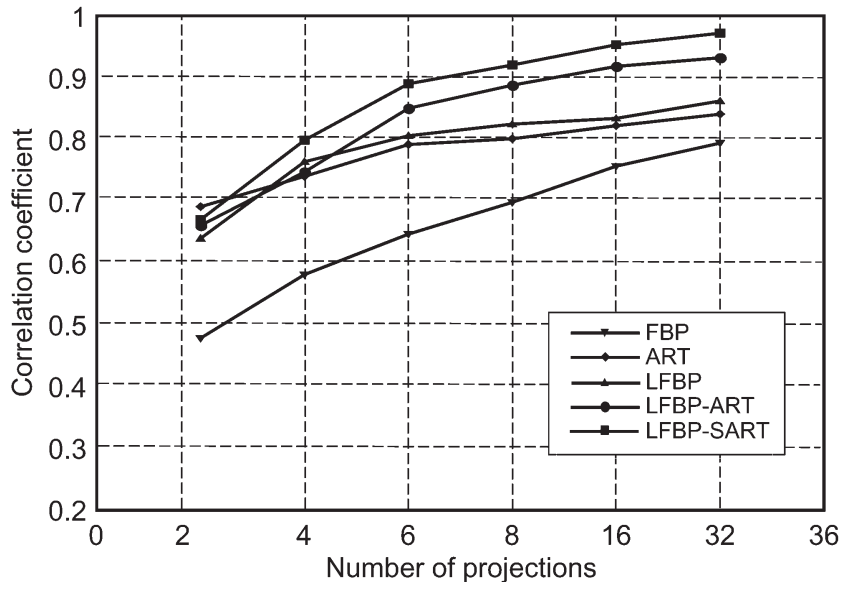

Fig. 8. CC for different projections.

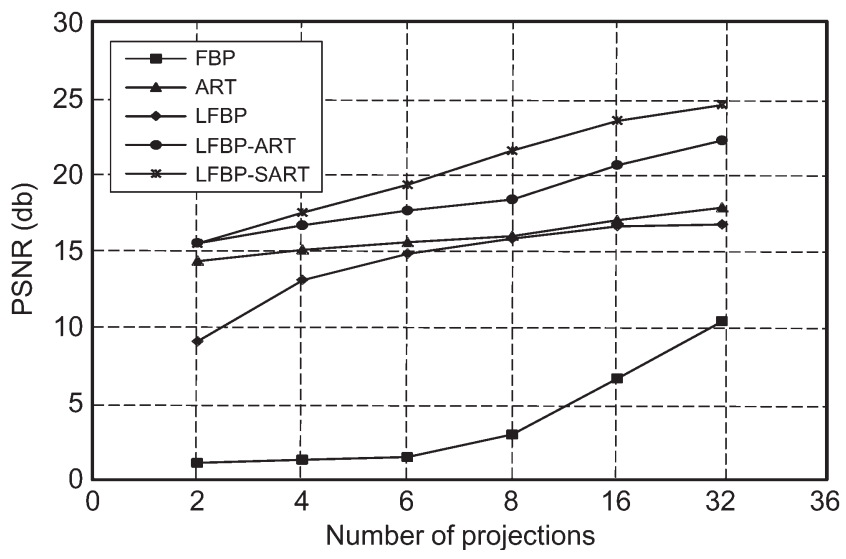

Fig. 9. PSNR for different projections.

the simulation results that the LFBP-SART reconstruction with eight projections gives a higher CC (by about 6\%) and an increased PSNR (more than 12\%) as compared to LFBP-ART algorithms with eight projections.

The CC and the PSNR between the original templates and the reconstructed results for eight projects were also computed for further comparison, and the results are summarized in Table II. It is evident that the LFBP-SART consistently gives a better CC and the highest PSNR in all cases. This investigation has led to the conclusion that an LFBP-SART with eight projections offers the best tomographic approach to the reconstruction problem. 
TABLE II

CC AND PSNR FOR DIFFERENT TOMOGRAPHIC ALGORITHMS

\begin{tabular}{|c|c|c|c|c|c|c|c|c|c|c|}
\hline \multirow[b]{2}{*}{ Template } & \multicolumn{2}{|c|}{ FBP } & \multicolumn{2}{|c|}{ LFBP } & \multicolumn{2}{|c|}{ ART } & \multicolumn{2}{|c|}{ LFBP-ART } & \multicolumn{2}{|c|}{ LFBP-SART } \\
\hline & $\mathrm{CC}$ & $\begin{array}{l}\text { PSNR } \\
(\mathrm{db})\end{array}$ & $\mathrm{CC}$ & $\begin{array}{c}\text { PSNR } \\
(\mathrm{db})\end{array}$ & $\mathrm{CC}$ & $\begin{array}{c}\text { PSNR } \\
(\mathrm{db})\end{array}$ & $\mathrm{CC}$ & $\begin{array}{c}\text { PSNR } \\
(\mathrm{db})\end{array}$ & $\mathrm{CC}$ & $\begin{array}{l}\text { PSNR } \\
(\mathrm{db})\end{array}$ \\
\hline $\begin{array}{c}\text { Head } \\
\text { Phantom }\end{array}$ & 0.70 & 2.96 & 0.82 & 15.79 & 0.80 & 15.96 & 0.88 & 18.34 & 0.93 & 20.74 \\
\hline Cross & 0.79 & 4.79 & 0.96 & 11.52 & 0.90 & 17.69 & 0.92 & 18.60 & 0.98 & 27.55 \\
\hline $\begin{array}{l}\text { Matrix burner } \\
\text { flames }\end{array}$ & 0.63 & 3.67 & 0.84 & 13.27 & 0.82 & 15.46 & 0.91 & 20.30 & 0.94 & 21.54 \\
\hline
\end{tabular}

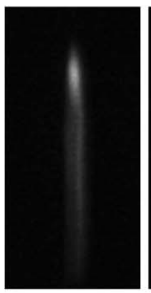

$0^{\circ}$

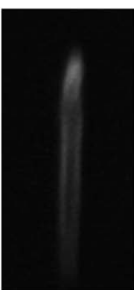

$22.5^{\circ}$

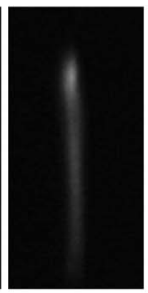

$45^{\circ}$

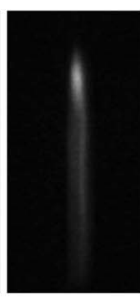

$67.5^{\circ}$

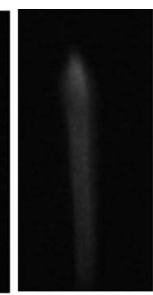

$90^{\circ}$

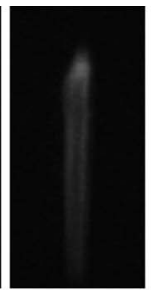

$112.5^{\circ}$

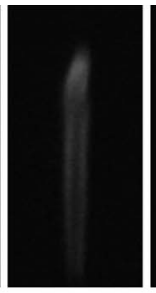

$135^{\circ}$

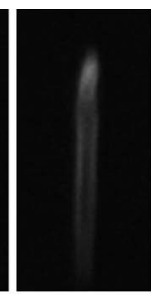

$157.5^{\circ}$

Fig. 10. Two-dimensional flame images taken using the eight imaging fiber bundles.

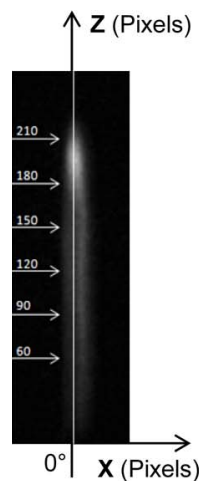

(a)

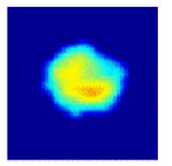

Section 60

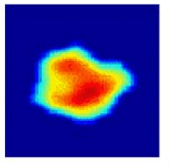

Section 150

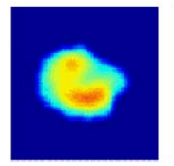

Section 90

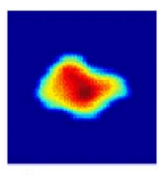

(b)

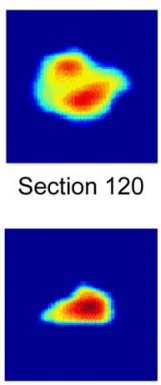

Section 210

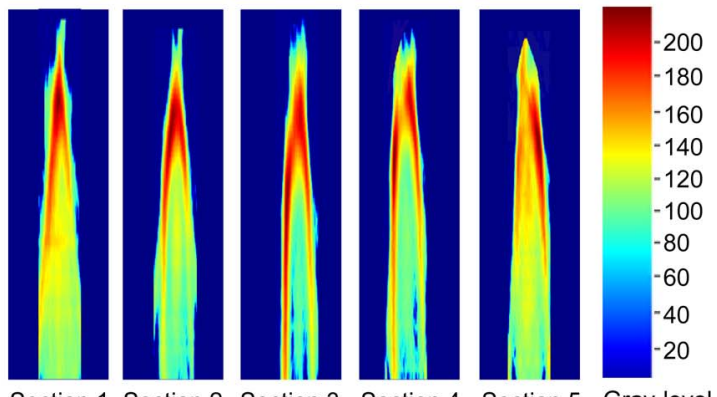

Section 1 Section 2 Section 3 Section 4 Section 5 Gray-level

(c)

Fig. 11. Luminosity reconstruction of flame cross- and longitudinal-sections. (a) Two-dimensional image. (b) Cross-sections. (c) Longitudinal-sections.

\section{B. Experimental Results}

To evaluate the performance of the developed system, experiments were conducted on a laboratory-scale gas-fired combustion test rig. In this paper, a burner with an outlet diameter of $20 \mathrm{~mm}$ was used to generate a non-premixed flame with an approximate height of up to $200 \mathrm{~mm}$ under a steady combustion condition. A mesh screen was mounted across the outlet of the burner to ensure a uniform velocity profile of the flow, and the Reynolds number of the flow was approximately 171 . The imaging system was used to capture 2-D images of the flame concurrently from eight different directions. Fig. 10 shows a typical set of the 2-D flame images which have a flame area of approximately $10 \mathrm{k}$ pixels.

The cross- and longitudinal-sections of the flame were reconstructed from the images obtained based on the LFBP-SART algorithm. Fig. 11 shows the luminosity reconstruction of flame cross- and longitudinal-sections. The pseudo-color technique is used here for a better data presentation, where the dark red represents the greatest gray-level and the dark blue represents the lowest gray-level. As can be seen, the reconstructions have indicated the variations of the gray-levels on the different crosssections. Comparing the reconstructed cross-sections with the original 2-D images, one can make several observations. For instance, cross-section 60 [Fig. 11(b)] is more circular as it is nearest to the burner outlet. Moving along the $Z$-axis, one can observe that the circularity of the cross-section degrades. This characteristic is also evident in the original 2-D images where different widths of the corresponding sections are evident. The luminosity distribution of the upper cross-section appears to have a non-circular outer shape.

The reconstruction of the flame cross-section can be computed repeatedly for a chosen pixel row along the $Z$-axis. The data obtained can also be used to reconstruct the longitudinalsections of the flame, as shown in Fig. 11(c). It should be noted 
that the visual representation of the reconstructed longitudinalsections depends on the point of view. The results shown in Fig. 11(c) refer to sections viewed from the direction $0^{\circ}$ (refer to Fig. 1). Section 3 depicts the longitudinal-section along the burner axis, while sections 1 and 2 present sections four and eight steps (pixels) back off the burner axis, and sections 4 and 5 present sections four and eight steps (pixels) forward the burner axis. Since sections 2, 3, and 4 are close to the burner axis, similar luminous profiles have been found, and they agree well with the original 2-D image of the flame [Fig. 11(a)].

\section{CONCLUSION}

An optical fiber and CCD camera based tomographic system for the 3-D reconstruction of flames has been designed and implemented. The system, which works on eight imaging fiber bundles coupled with two RGB CCD cameras, is capable of capturing 2-D images of a flame concurrently from eight different directions around the flame so that improved system simplicity and spatial resolution are achieved. The characteristic evaluation of the imaging fiber bundles has been conducted using an LED white backlight panel. The eight imaging fiber bundles have very similar intensities, with the maximum discrepancy no greater than 2 gray-levels. The system is calibrated using a circular template with several adjacent reference marks, and the maximum deviation of the measured length from the reference value is no more than $2 \%$. The radial distortion and vignetting effect of the imaging system have also been investigated. The normalized radial error that was found is not greater than $2 \%$, where the normalized standard deviation is less than $4 \%$ for the objective distance of $250 \mathrm{~mm}$. A new approach of combining the LFBP and SART (i.e., LFBP-SART) to the reconstruction of flame sections has been proposed and evaluated. Simulation results have demonstrated that, for eight image projections provided by the image sensors, the proposed LFBP-SART approach consistently gives a better CC and the highest PSNR for the given templates. The experimental results obtained using the flames have shown that the optical sensing arrangement and the developed tomographic algorithm are effective in the acquisition of the flame images and the subsequent reconstruction of the luminosity distribution of the cross- and longitudinal-sections of the flame from eight image projections.

\section{REFERENCES}

[1] Y. Yan, T. Qiu, G. Lu, M. M. Hossain, and G. Gilabert, "Recent advances in 3D flame tomography," in Proc. 6th World Congr. Ind. Process Tomogr., Beijing, China, Sep. 6-9, 2010, pp. 1530-1539.

[2] J. Ballester and T. Garcia-Armingol, "Diagnostic techniques for the monitoring and control of practical flames," Progr. Energy Combust. Sci., vol. 36, no. 4, pp. 375-411, 2010.

[3] J. Nygren, J. Hult, M. Richter, M. Aldén, M. Christensen, A. Hultqvist, and B. Johansson, "Three-dimensional laser induced fluorescence of fuel distribution in an HCCI engine," Proc. Combust. Inst., vol. 29, no. 1, pp. 679-685, 2002.

[4] B. Böhm, C. Kittler, A. Nauert, and A. Dreizler, "Diagnostics at high repetition rates: New insights into transient combustion phenomena," in Proc. 3rd Eur. Combust. Meet., Crete, Greece, Apr. 11-13, 2007, 5 pp., Section 5-1.

[5] E. D. Torniainen, A. K. Hinz, and F. C. Gouldin, "Tomographic analysis of unsteady, reacting flows: Numerical investigation," AIAA J., vol. 36, no. 7 , pp. 1270-1278, Jul. 1998.
[6] W. Fisher and H. Burkhardt, "Three-dimensional temperature measurement in flames by multispectral tomographic image analysis," in Proc. SPIE, 1990, vol. 1349, pp. 96-105.

[7] P. M. Brisley, G. Lu, Y. Yan, and S. Cornwell, "Three dimensional temperature measurement of combustion flames using a single monochromatic CCD camera," IEEE Trans. Instrum. Meas., vol. 54, no. 4, pp. 1417-1421, Aug. 2005.

[8] Q. Huang, F. Wang, D. Liu, Z. Ma, J. Yan, Y. Chi, and K. Cen, "Reconstruction of soot temperature and volume fraction profiles of an asymmetric flame using stereoscopic tomography," Combust. Flame, vol. 156, no. 3, pp. 565-573, Mar. 2009.

[9] B. Zhou, S. Wang, C. Xu, and J. Zhang, "3-D flame temperature reconstruction in optical sectioning tomography," in Proc. Int. Workshop Imag. Syst. Tech., Shenzhen, China, May 11-12, 2009, pp. 313-318.

[10] H. C. Bheemul, G. Lu, and Y. Yan, "Three-dimensional visualization and quantitative characterization of gaseous flames," Meas. Sci. Technol., vol. 13 , no. 10 , pp. 1643-1650, Oct. 2002.

[11] Y. Ishino, T. Hirano, M. Hirano, and N. Ohiwa, "Non-scanning 3D-CT visualizations of premixed flames with a 40 lens camera," in Proc. 6th Pacific Symp. Flow Vis. Image Process., HI, May 16-19, 2007, vol. A2-2, pp. 1-6.

[12] Y. Ishino and N. Ohiwa, "Three-dimensional computerized tomographic reconstruction of instantaneous distribution of chemiluminescence of turbulent premixed flame," JSME Int. J., vol. 48, no. 1, pp. 34-40, 2005, Series B.

[13] H. C. Zhou, C. Lou, Q. Cheng, Z. Jiang, J. He, B. Huang, Z. Pei, and $\mathrm{C}$. $\mathrm{Lu}$, "Experimental investigations on visualization of threedimensional temperature distributions in a large-scale pulverized-coalfired boiler furnace," Proc. Combust. Inst., vol. 30, no. 1, pp. 1699-1706, Jan. 2005

[14] J. Floyd and A. M. Kempf, "Computed tomography of chemiluminescence (CTC): High resolution and instantaneous 3D measurement of a matrix burner," Proc. Combust. Inst., vol. 33, no. 1, pp. 751-758, 2011.

[15] J. Floyd and A. M. Kempf, "Computed tomography of chemiluminescence (CTC): Instantaneous 3D measurement and phantom studies of a turbulent opposed jet flame," Combust. Flame, vol. 158, no. 2, pp. 376391, Feb. 2011.

[16] N. Anikin, R. Suntz, and H. Bockhorn, "Tomographic reconstruction of the $\mathrm{OH}^{*}$-chemiluminescence distribution in premixed and diffusion flames," Appl. Phys. B, Lasers Opt., vol. 100, no. 3, pp. 675-694, Sep. 2010.

[17] A. Schwarz, "Multi-tomographic flame analysis with a Schlieren apparatus," Meas. Sci. Technol., vol. 7, no. 3, pp. 406-413, Mar. 1996.

[18] X. Wan, W. Xiong, Z. Zhang, and F. Chang, "An online emission spectral tomography system with digital signal processor," Opt. Exp., vol. 17, no. 7, pp. 5279-5284, Mar. 2009.

[19] I. Ihrke and M. Magnor, "Image-based tomographic reconstruction of flames," in Proc. Eurographics/ACM SIGGRAPH Symp. Comp. Animat., Max-Planck-Institut für Informatik, Saarbrücken, Germany, 2004, pp. $365-373$.

[20] F. Moratti, M. Annunzotia, and S. Giammartini, An Artificial Vision System for the 3D Reconstruction and the Dynamical Characterisation of Industrial Flames. Rome, Italy: ENEA-C.R.E. Casaccia, 1997.

[21] G. Gilabert, G. Lu, and Y. Yan, “Three-dimensional tomographic reconstruction of luminosity distribution of a combustion flame," IEEE Trans. Instrum. Meas., vol. 56, no. 4, pp. 1300-1306, Aug. 2007.

[22] M. M. Hossain, G. Lu, and Y. Yan, "Three-dimensional reconstruction of combustion flames through optical fiber sensing and CCD imaging," in Proc. IEEE I2MTC, Hangzhou, China, May 10-12, 2011, pp. 79-83.

[23] A. C. Kak and M. Slaney, Principles of Computerized Tomographic Imaging. Piscataway, NJ: IEEE Press, 1988.

[24] W. Yang and H. Peng, "Image reconstruction algorithms for electrical capacitance tomography," Meas. Sci. Technol., vol. 14, no. 1, pp. R1-R13, Jan. 2003

[25] L. J. Xu and L. A. Xu, "Ultrasound tomography system used for monitoring bubbly gas/liquid two-phase flow," IEEE Trans. Ultrason., Ferroelectr., Freq. Control, vol. 44, no. 1, pp. 67-76, Jan. 1997.

[26] G. N. Hounsfield, "A method and apparatus for examination of body by radiation such as X or gamma radiation," U.K. Patent 1283915 , Aug. 2, 1972.

[27] D. Raparia, J. Alessi, and A. Kponou, "The algebriac reconstruction technique," in Proc. Particle Accelerator Conf., Vancouver, B.C., Canada, May 12-16, 1997, pp. 2023-2025.

[28] M. Jiang and G. Wang, "Convergence studies on iterative algorithms for image reconstruction," IEEE Trans. Med. Imag., vol. 22, no. 5, pp. 367 375, May 2003 


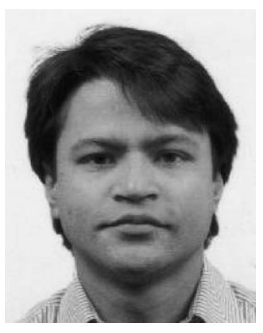

Md. Moinul Hossain (M'11) received the B.Eng. degree in computer science and engineering from International Islamic University, Chittagong, Bangladesh, in 2004 and the M.Sc. degree in wireless communication and system engineering from the University of Greenwich, U.K., in 2008. He is currently working toward the Ph.D. degree in the Instrumentation, Control and Embedded Systems Research Group, School of Engineering and Digital Arts, University of Kent, Canterbury, U.K.

His research interest includes vision-based instrumentation systems and measurements, digital image processing, and computed tomographic techniques.

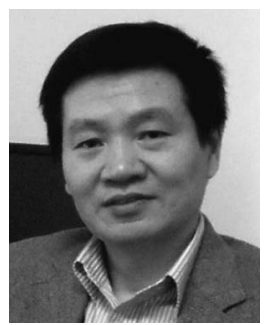

Gang Lu (SM'05) received the B.Eng. degree in mechanical engineering from Central South University, Changsha, China, in 1982 and the Ph.D. degree in advanced combustion instrumentation from the University of Greenwich, London, U.K., in 2000.

$\mathrm{He}$ started his career as a research engineer and worked on mechanical design and engineering development for ironmaking and steelmaking industries in China for more than ten years. From 2000 to 2006, he worked as a Postdoctoral Research Fellow with the University of Greenwich and the University of Kent, Canterbury, U.K. He is currently a Lecturer in electronics and instrumentation with the School of Engineering and Digital Arts, University of Kent. He has been involved in a range of projects on advanced monitoring and characterization of fossil fuel flames. His main research interests include combustion instrumentation.

Dr. Lu is a Chartered Engineer and a member of the Energy Institute. He was the recipient of the Engineering Innovation Prize by the Institution of Engineering Technology in 2006.

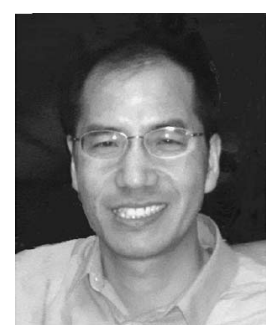

Yong Yan (M'04-SM'04-F'11) received the B.Eng. and M.Sc. degrees in instrumentation and control engineering from Tsinghua University, Beijing, China, in 1985 and 1988, respectively, and the Ph.D. degree in solids flow measurement and instrumentation from the University of Teesside, Middlesbrough, U.K., in 1992.

In 1988, he started his academic career as an Assistant Lecturer with Tsinghua University. In 1989 he was with the University of Teesside as a Research Assistant. In 1993-1996, after a short period of postdoctoral research, he worked initially as a Lecturer with the University of Teesside and then, in 1996-2004, as a Senior Lecturer, Reader, and Professor with the University of Greenwich, London, U.K. He is currently a Professor in electronic instrumentation; the Head of the Instrumentation, Control and Embedded Systems Research Group; and the Director of Research with the School of Engineering and Digital Arts, University of Kent, Canterbury, U.K. He has published in excess of 270 research papers in journals and conference proceedings, in addition to 12 research monographs.

Dr. Yan is a fellow of the Institution of Engineering Technology (IET) [formerly Institution of Electrical Engineers (IEE)], the Institute of Physics, and the Institute of Measurement and Control. In recognition of his contributions in pulverized fuel flow metering and combustion flame imaging, he was awarded the Achievement Medal by the IEE in 2003, the Engineering Innovation Prize by the IET in 2006, and the Rushlight Commendation Award in 2009. 